\title{
Development of an all PM mode lock fiber laser for space borne frequency reference
}

Yuichi Takeuchi, Ryota Saito, Shun Endo, Kana Matsusaka, Mitsuru Musha

Yuichi Takeuchi, Ryota Saito, Shun Endo, Kana Matsusaka, Mitsuru Musha, "Development of an all PM mode lock fiber laser for space borne frequency reference," Proc. SPIE 11852, International Conference on Space Optics ICSO 2020, 1185230 (11 June 2021); doi: 10.1117/12.2599616

EDIE Event: International Conference on Space Optics - ICSO 2021, 2021, Online Only 


\section{International Conference on Space Optics-ICSO 2020}

Virtual Conference

30 March-2 April 2021

Edited by Bruno Cugny, Zoran Sodnik, and Nikos Karafolas
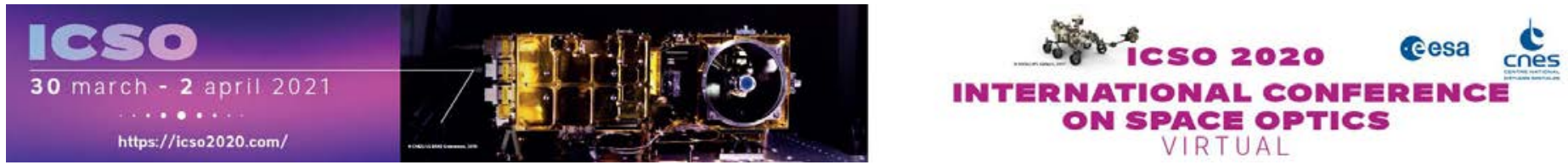

\section{Development of an all PM mode lock fiber laser for space borne frequency reference}

\section{Cesa tsopenestums ecnes}




\title{
Development of an all PM mode lock fiber laser for space borne frequency reference
}

\author{
Yuichi Takeuchi $^{1 *}$, Ryota Saito ${ }^{1}$, Shun Endo ${ }^{1}$, Kana Matsusaka ${ }^{1}$, and Mitsuru Musha ${ }^{1}$ \\ ${ }^{1}$ Institute for Laser Science, University of Electro-Communications (ILS/UEC), 1-5-1 \\ Chofugaoka, Chofu-shi, Tokyo, JAPAN
}

\begin{abstract}
For the next-generation Japanese global navigation satellite system, we have developed the space borne highlystable microwave generation system by combination of an optical frequency reference and an optical frequency comb. A 1030-nm iodine-stabilized laser has been developed as the optical frequency reference whose frequency stability reaches $10^{-14}$ level at the averaging time of 2000 seconds. We have developed a figure- 8 type polarization maintaining (PM) fiber mode-locked laser as a light source for the space borne optical frequency comb. The repetition frequency and the center wavelength of our mode-locked fiber laser are $48.7 \mathrm{MHz}$ and $1560 \mathrm{~nm}$, respectively, and we demonstrated a continuous operation of its mode-locking for more than 1 week. Also, three kinds of thermal tests have been applied to our mode-locked laser with the temperature range from 5 to 60 Celsius, and the stable operation without breaking mode-locking has been confirmed. Furthermore, we have analyzed the results of a thermal cycling test. The temporal variation of the optical length in the fiber is mainly affected by that of the metal plate which is 11.8 times stronger than that of the surrounding air.
\end{abstract}

Keywords: space borne frequency reference, mode-locked fiber laser, nonlinear amplifying loop mirror, optical frequency comb, highly-stable microwave generation, iodine-stabilized laser

\section{INTRODUCTION}

Space borne frequency references have extensive applications in metrology, astronomy, remote sensing, space gravitational wave detection, and global navigation satellite positioning system (GNSS). Especially, GNSS has been widely used as the indispensable social infrastructure in recent years. In order to improve the position accuracy, we have promoted next-generation Japanese GNSS which consists of a small number of quasi-zenith satellites. A space borne frequency reference is one of the essential devices for improving the accuracy of the positioning. Currently, the rubidium atomic clock is used for the space borne frequency reference whose frequency stability is limited to the $10^{-12}$ levels. Therefore, we have proposed the optical-based highly-stable microwave generation system by using a combination of an optical frequency reference and an optical frequency comb. Since the phase sensitivity of the optical frequency is much higher than that of the microwave frequency, the optical frequency stability is expected to reach $10^{-15}$ or higher levels by the precision frequency and phase control. Though the frequency stability of the optical lattice clock has reached $10^{-18}$ level in the laboratory environment, ${ }^{1}$ it is too complicated to operate in the satellite. The iodine-stabilized laser, on the other hand, is relatively compact and simple system whose frequency stability reaches $10^{-15}$ levels, and the space borne iodine-stabilized laser has been developed. ${ }^{2}$ From the numerical simulation, the $10^{-15}$ levels frequency reference in GNSS would improve the positioning accuracy by a few orders of magnitudes. ${ }^{3}$ The requirements of the space borne optical frequency reference for Japanese next-generation GNSS are listed in Table 1.

Table 1. The requirements for the space borne optical frequency reference.

\begin{tabular}{c|c|c} 
Microwave frequency & Frequency stability & Operation lifetime \\
\hline $51.15 \mathrm{MHz}$ & $<10^{-14}$ & $>10$ years
\end{tabular}

Further author information: (Send correspondence to Yuichi Takeuchi)

Yuichi Takeuchi: E-mail: y_takeuchi@ils.uec.ac.jp, Telephone: +81 424435711 


\section{OPTICAL-BASED HIGHLY-STABLE MICROWAVE GENERATION SYSTEM}

Our optical-based highly-stable microwave generation system consists of an optical frequency reference and an optical frequency comb. We choose an iodine-stabilized laser as an optical frequency reference because it would keep its high frequency stability even in space. In addition, a fiber-based mode-locked laser is selected as the frequency down-converter because of its robustness (Figure 1). The details of the mode-locked laser are described in Section 3.

$\star$ Optical frequency reference
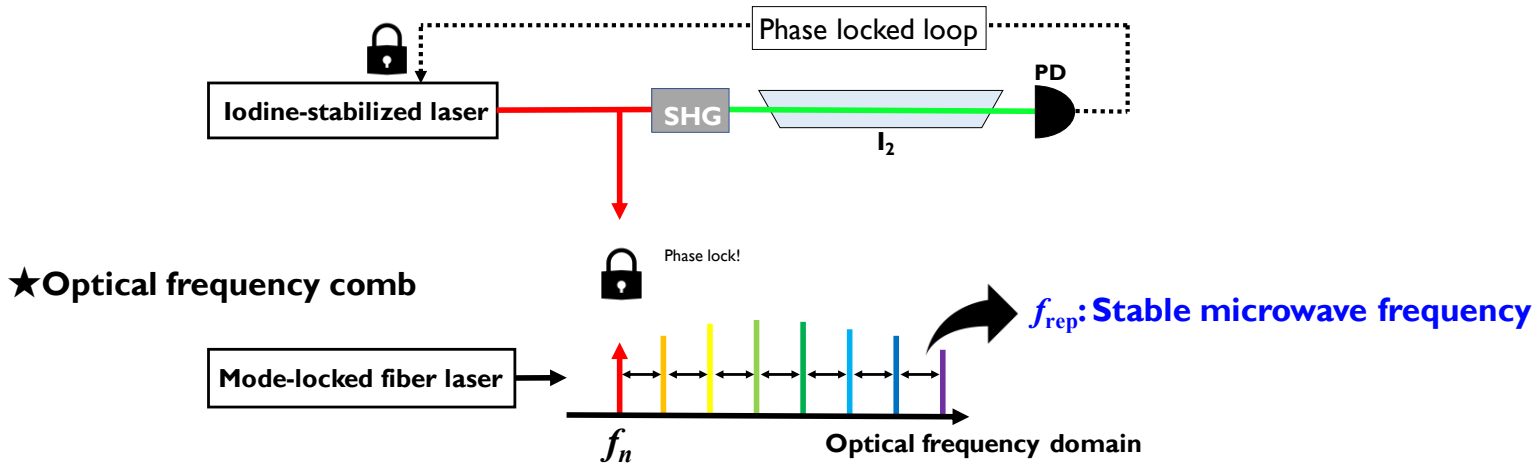

Figure 1. The schematics of the optical-based highly-stable microwave generation system.

\subsection{Iodine-stabilized laser}

The iodine-stabilized lasers have been developed for a light source of the space gravitational wave detection project in Japan named DECIGO. Hyperfine components of the iodine molecules at 515 nm is used as a frequency reference. The 515-nm light source is generated from the second harmonics of the 1030 nm narrow-linewidth fiber distributed feedback (DFB) laser. We have reported the details of the breadboard model of our iodine-stabilized laser at ICSO2018, ${ }^{4}$ whose frequency stability is $10^{-14}$ level at the averaging time of 2000 seconds, and all the optical components are mounted on a $550 \mathrm{~mm} \times 300 \mathrm{~mm}$ aluminum breadboard (Figure 2).

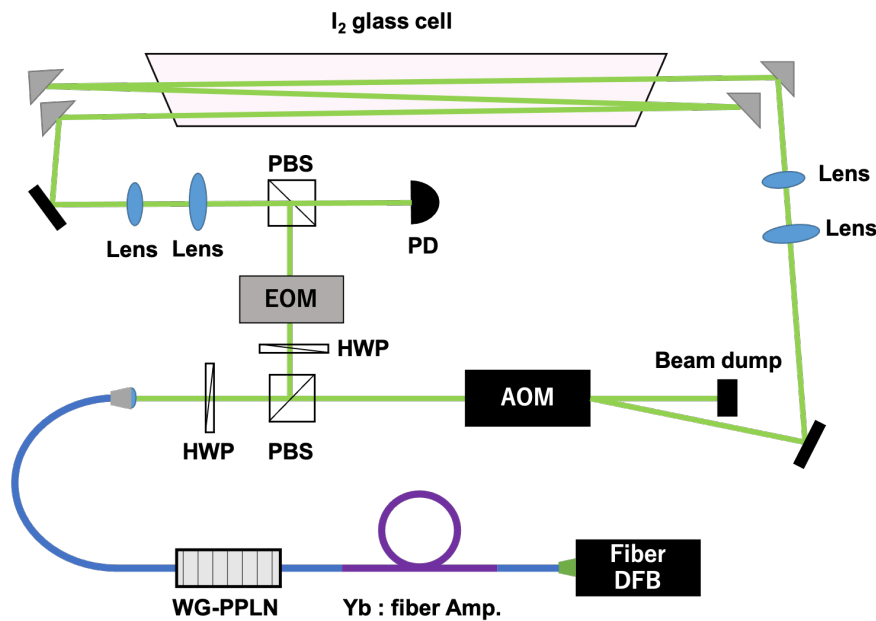

Figure 2. The schematic diagram of our iodine-stabilized laser of the breadboard model. 


\subsection{Fiber-based optical frequency comb}

With the advent of the mode-locked laser based optical frequency combs in the $2000 \mathrm{~s}^{5,6}$, the optical frequency is directly connected to the microwave frequency. The optical frequency comb is composed of equally-spaced frequency modes which becomes a ruler in the optical frequency domain. The frequency of $n$th mode of the optical frequency comb is written as

$$
f_{n}=f_{\text {ceo }}+n f_{\text {rep }},
$$

where $f_{\text {rep }}$ and $f_{\text {ceo }}$ are the repetition rate frequency and the carrier-envelope offset frequency in the microwave domain, respectively. When the $f_{n}$ is phase locked to the optical frequency reference (and $f_{\text {ceo }}$ is also locked to the microwave frequency reference), the stable microwave signal can be obtained as $f_{\text {rep }}$ whose frequency stability is almost the same as that of the optical frequency reference. As a consequence, a highly-stable frequency reference in the optical frequency region is down-converted to the microwave region without degradation. ${ }^{7}$

\section{ALL-PM MODE-LOCKED FIBER LASER}

As a light source for our optical frequency comb, a fiber-based mode-locked laser is chosen because of its light weight, compactness and its alignment-free configuration whose size and robustness are suitable for the space borne system. The mode-locked laser is a kind of ultra-short pulse lasers. For mode-locking operation, the intensity-dependent loss mechanism is necessary which is called as mode locker. Nonlinear polarization rotation (NPR) is widely used as a mode locker for fiber-based optical frequency comb. The polarization state evolution of the optical pulse in the fiber depends on its peak intensity, and the stronger pulse is selected by using a polarizer in the cavity to make mode-locking. NPR can generate ultra-short duration pulse train with excellent low phase noise, which is suitable for an optical frequency comb. However, NPR has a problem of its low robustness because the polarization state in the fiber is easily disturbed by the external perturbations such as the temperature variations or the mechanical vibrations. Therefore, we have selected another mode-locking mechanism called nonlinear amplifying loop mirror (NALM) for our mode-locked laser. ${ }^{8}$ Since NALM is a polarization-independent mechanism, it is robust against the external disturbance. NALM is a kind of the Sagnac interferometer with intensity-dependent optical switch mechanism in which anti-symmetric nonlinear effects in the fiber loop causes phase difference between CW and CCW rotated pulses. ${ }^{9}$ Our figure- 8 mode-locked laser consists of NALM and another fiber loop with the optical isolator, and consequently it has intensity-dependent optical loss which acts as a mode locker. We have developed the erbium-doped fiber (EDF) mode-locked laser at $1.5 \mu \mathrm{m}$ whose spectral range can be expanded from $1 \mu \mathrm{m}$ to $2 \mu \mathrm{m}$ by using highly nonlinear fiber (HNLF) ${ }^{10}$ so that the optical modes at longer end can be phase locked to the iodine-stabilized fiber DFB laser at $1.03 \mu \mathrm{m}$.

\subsection{All-PM figure-8 mode-locked laser}

Figure 3 shows the schematics of our mode-locked laser. The oscillator is an all-PM fiber figure- 8 configuration. 


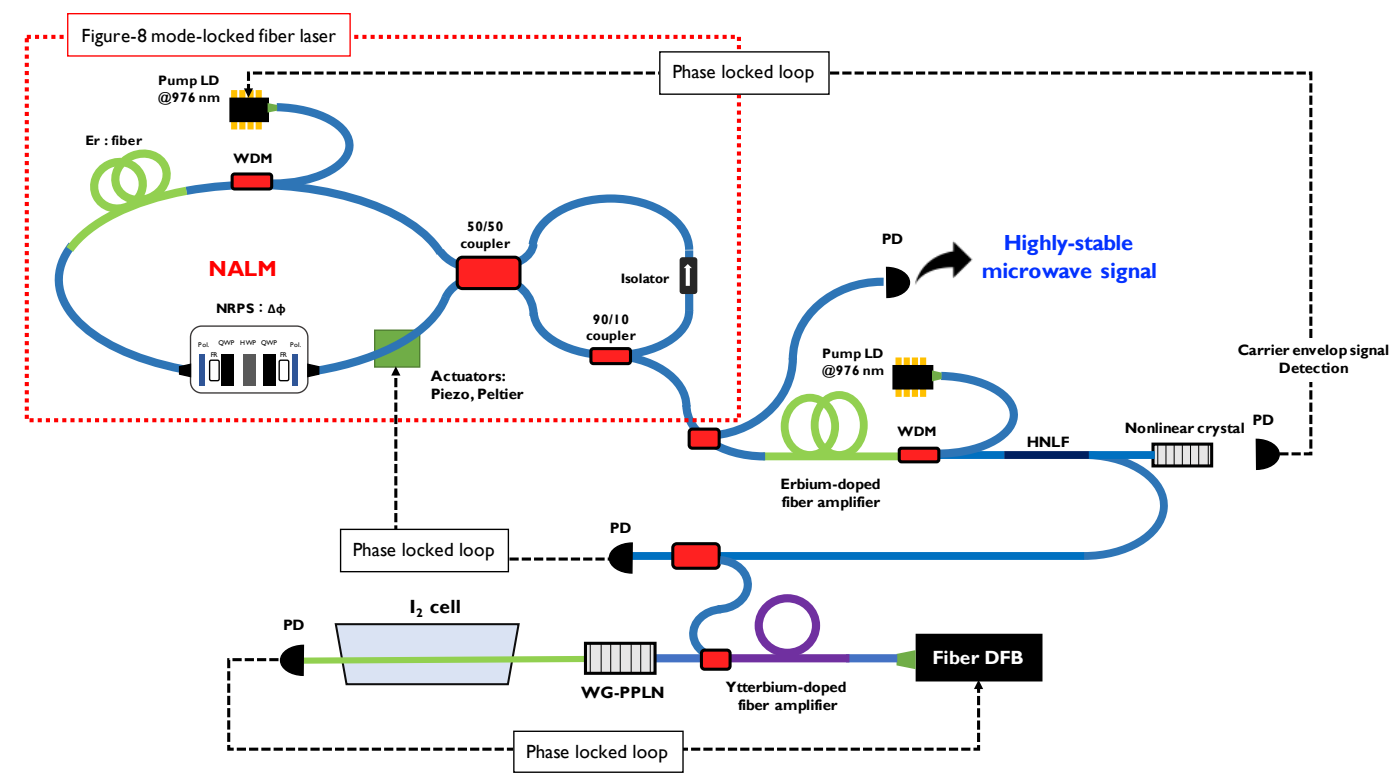

Figure 3. The schematic diagram of the our figure-8 mode-locked laser system. LD: laser diode, WDM: wavelength-division multiplexer, HNLF: highly nonlinear fiber, WG-PPLN: waveguide periodically poled lithium niobate, PD: photodetector.

In principle, the phase noise of the mode-locked laser decreases as the net dispersion of the cavity approaches to zero, which is called as the stretched pulse region. ${ }^{11}$ In order to improve the phase noise of our mode-locked laser, the net dispersion of the cavity is designed to be $0.005 \mathrm{ps}^{2}$ in the stretched-pulse region. Our mode-locked laser obtains high-energy pulses with a smooth stretched-pulse spectrum without any additional peaks like Kelly sidebands (Figure 4).

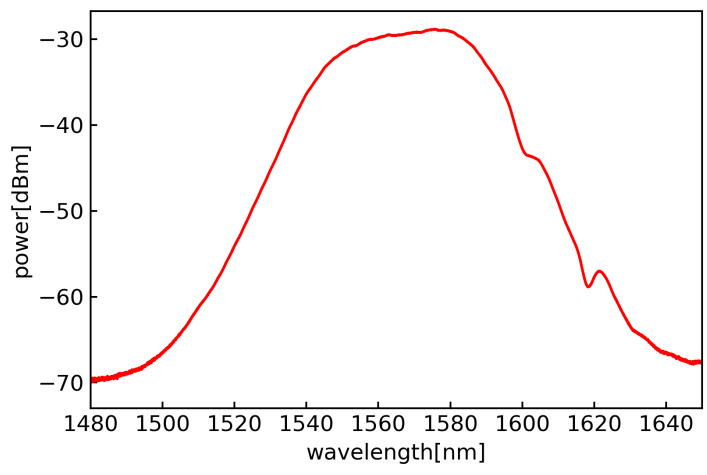

(a) The optical spectrum in the stretched-pulse region

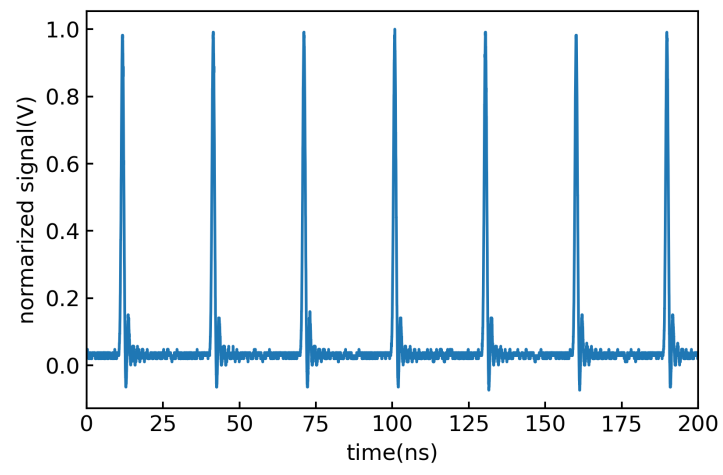

(b) The pulse train of the mode-locking

Figure 4. The time waveform of the figure- 8 mode-locked laser. The time internal of the pulse train is $20.5 \mathrm{~ns}$.

With the help of a nonreciprocal phase shifter (NRPS), the cavity length of the NALM becomes shorter to increase $f_{\text {rep }}$ frequency up to $48.7 \mathrm{MHz}$ which is close to the requirement frequency of $51.15 \mathrm{MHz}$. The average power and the center wavelength of our mode-locked laser are $0.8 \mathrm{~mW}$ and $1560 \mathrm{~nm}$, respectively. The output power of the mode-locked laser is amplified by using EDF amplifier, and its amplified output is introduce into HNLF to expand the mode spectrum. Figure 5 shows the expanded spectrum of the mode-locked laser. Red trace in Figure 5 is experimental results of the octave-spanning spectrum. 


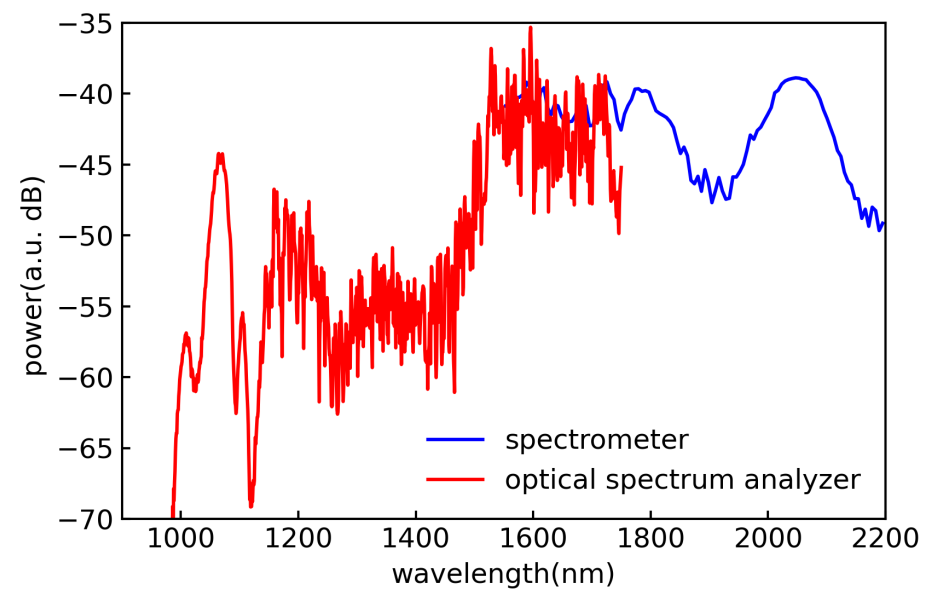

Figure 5. The octave-spanning spectrum.

\section{CONTINUOUS OPERATION TEST AND THERMAL TEST}

We have investigated the robustness of our mode-locked laser against the environmental perturbations. The temperature of the surrounding air and a metal plate are controlled. All fiber components are fixed on the metal plate. First, the long-term continuous operation is tested in the laboratory environment, and the stable continuous operation for more than one week is confirmed, where the continuous operation is forced to stop at the end of the experiment. Next, our mode-locked laser is tested under various temperature conditions between 5 and 60 Celsius to confirm its operation temperature range. Our mode-locked laser is contained in a constant temperature chamber, and the thermal tests are conducted under following three temperature conditions.

1. Constant temperature from 5 to 60 Celsius in every 10 degree

2. Slow cyclic temperature variation

3. Steep temperature change (heat shock test)

In all cases, our mode-locked laser shows the stable continuous operation without breaking the mode-locked operation even in the heat shock test. In the heat shock test, the steep temperature change is applied to a small part of metal base plate. Although the $f_{\text {rep }}$ follows its steep temperature change, the laser keeps mode-locking (Figure 6). 


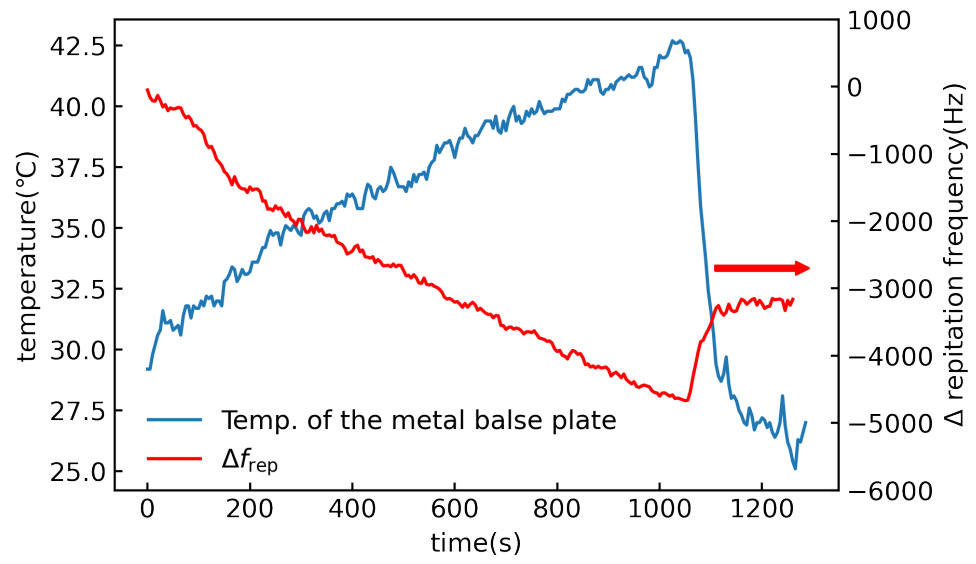

Figure 6 . The results of the heat shock test. Red and blue traces indicate temporal variations of the $\Delta f_{\text {rep }}$ and the metal base plate, respectively.

From these temperature tests, it is concluded that the figure- 8 type mode-locked laser would perform the stable operation under typical satellite temperature conditions. We also analyzed the results of the cyclic temperature test to investigate the dependence of the laser cavity length on the temperature of surrounding air and of the base plate. The repetition frequency of the mode-locked laser, $f_{\text {rep }}$ is inversely proportional to the cavity length. Figure 7 shows the temporal variations of the $f_{\text {rep }}$ (red traces) in the slow and fast cyclic temperature tests. Orange and blue traces indicate the temperature of the surrounding air and the base plate, respectively. In the slow cyclic test, the temperature of the air is changed from 5 to 60 Celsius with the period of 20000 seconds. The temperature of the air, in the fast cyclic test, is changed with the slope of 6 degree per minutes.

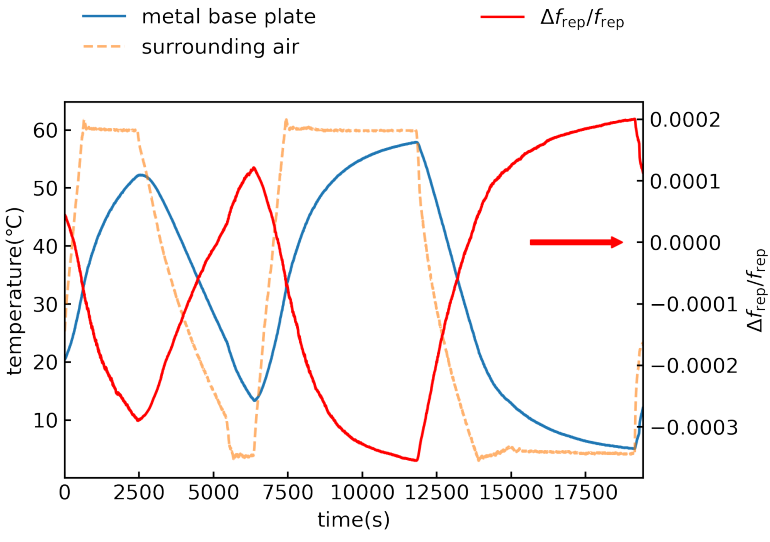

(a) Fast cyclic test

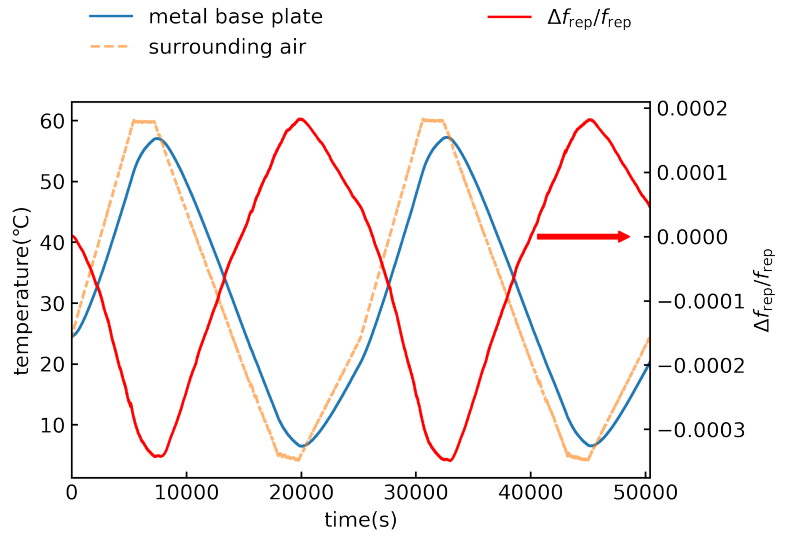

(b) Slow cyclic test

Figure 7. The time-series graphs are the cycling test results. Left graph shows slow cyclic thermal test (the period is 20000 seconds). Right graph shows fast cyclic thermal test (the slope of the temperature is 6 degrees per minutes).

The temporal variation of the $f_{\text {rep }}$ is applied to the following fitting equation for the multiple regression analysis where $T_{\text {metal }}$ and $T_{\text {air }}$ are the temperature of the base plate and the surrounding air, respectively.

$$
\frac{\Delta f_{\text {rep }}(t)}{f_{\text {rep }}}=-\frac{\delta L(t)}{L}=\alpha \frac{d S}{d T} d T_{\text {metal }}(t)+\beta \frac{d S}{d T} d T_{\text {air }}(t)+\text { err. }
$$

$d S / d T$ is the thermal expansion coefficient of the fiber optical length. The temperature of the base plate shows certain contribution to the $f_{\text {rep }}$, which is 11.8 times stronger than that of the surrounding air (Figure 
8). It is concluded that the $f_{\text {rep }}$ would be fully controlled by the temperature of the base plate against any temperature variations of the surrounding air in the satellite.

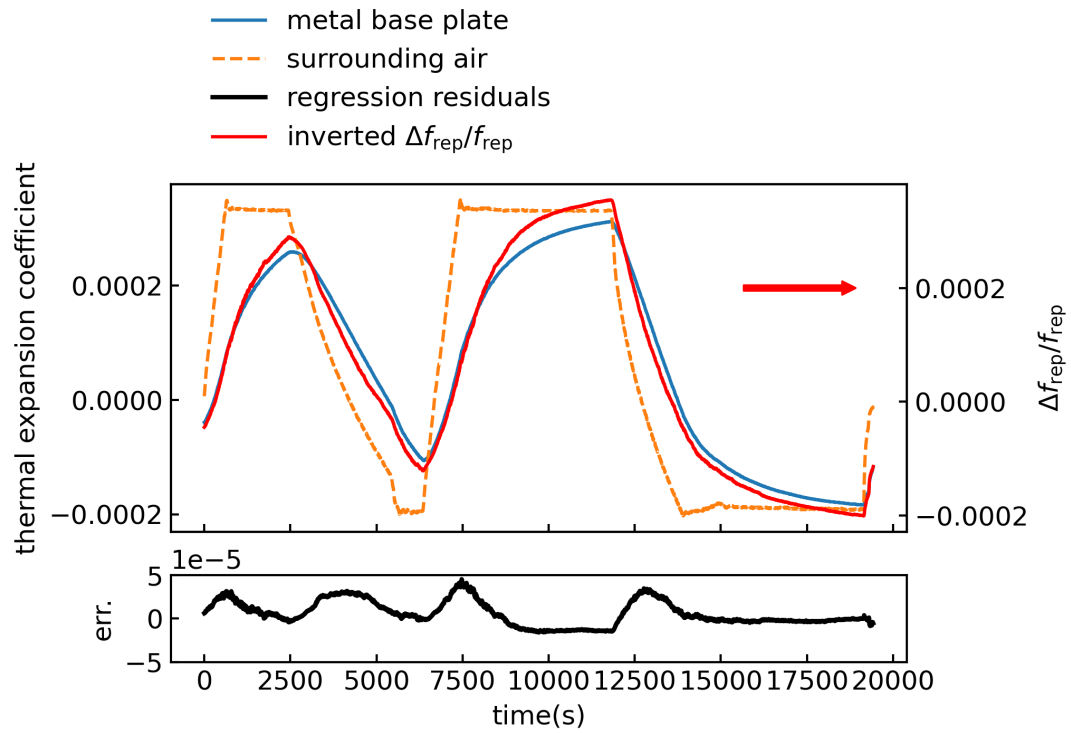

Figure 8. The multiple regression results of the fast cyclic test. The contribution parameter $\alpha, \beta$ and $\alpha / \beta$ are $-1.00,-0.08$ and 11.8 , respectively.

\section{CONCLUSIONS AND FUTURE PROSPECTS}

We propose a highly-stable microwave generation system in space by using an optical frequency reference and an optical frequency comb. For the light source of the optical frequency comb, we have developed the all-fiber figure- 8 mode-locked laser based on PM fiber. The repetition frequency, the average power, and the center wavelength are $48.7 \mathrm{MHz}, 0.8 \mathrm{~mW}, 1560 \mathrm{~nm}$. Our mode-locked laser shows stable continuous operation under three kinds of thermal conditions, and also the long-term operation for more than one week. We now investigate the phase noise of our figure- 8 mode-locked laser, which is indispensable for realizing the precision space borne optical frequency comb. Moreover, we will evaluate the frequency stability of the microwave signal generated from our system.

\section{ACKNOWLEDGMENTS}

This work was supported by the Japan Aerospace Exploration Agency (JAXA).

\section{REFERENCES}

[1] Takamoto, M., Hong, F.-L., Higashi, R., and Katori, H., "An optical lattice clock," Nature 435(7040), 321-324 (2005).

[2] Schuldt, T., Döringshoff, K., Kovalchuk, E. V., Keetman, A., Pahl, J., Peters, A., and Braxmaier, C., "Development of a compact optical absolute frequency reference for space with 10- 15 instability," Applied optics 56(4), 1101-1106 (2017).

[3] Matsushita, S., Takiguchi, H., Sasaki, T., Yamada, H., Kawano, I., Inoue, K., Takeuchi, Y., and Musha, M., "Simulation of the future qzss equipped the optical clock," in [Proceedings of the 33rd International Technical Meeting of the Satellite Division of The Institute of Navigation (ION GNSS+ 2020)], 1229-1237 (2020).

[4] Suemasa, A., Shimo-oku, A., Ohtsuka, S., Nakamori, M., and Musha, M., "Stable and high power 515nm lasers for the space gravitational wave detector: Decigo," in [International Conference on Space Optics-ICSO 2018], 11180, 111800V, International Society for Optics and Photonics (2019). 
[5] Hänsch, T. W., "Nobel lecture: passion for precision," Reviews of Modern Physics 78(4), 1297 (2006).

[6] Hall, J. L., "Nobel lecture: Defining and measuring optical frequencies," Reviews of Modern Physics 78(4), 1279 (2006).

[7] Fortier, T. M., Kirchner, M. S., Quinlan, F., Taylor, J., Bergquist, J., Rosenband, T., Lemke, N., Ludlow, A., Jiang, Y., Oates, C., et al., "Generation of ultrastable microwaves via optical frequency division," Nature Photonics 5(7), 425-429 (2011).

[8] Doran, N. and Wood, D., "Nonlinear-optical loop mirror," Optics letters 13(1), 56-58 (1988).

[9] Fermann, M. E., Haberl, F., Hofer, M., and Hochreiter, H., "Nonlinear amplifying loop mirror," Optics Letters 15(13), 752-754 (1990).

[10] Nicholson, J., Yan, M., Wisk, P., Fleming, J., DiMarcello, F., Monberg, E., Yablon, A., Jørgensen, C., and Veng, T., "All-fiber, octave-spanning supercontinuum," Optics letters 28(8), 643-645 (2003).

[11] Kim, J. and Song, Y., "Ultralow-noise mode-locked fiber lasers and frequency combs: principles, status, and applications," Advances in Optics and Photonics 8(3), 465-540 (2016). 